\title{
A PERCEPÇÃO DOS TRABALHADORES RURAIS SOBRE A AUTOEXPOSIÇÃO AOS AGROTÓXICOS
}

\author{
PERCEPTION OF RURAL WORKERS ON \\ AUTO EXPOSURE TO PESTICIDES
}

Jéssica Vilela Silva', Loraine Pires Vilela', Maiara Silva Moraes' ${ }^{1}$ Cristiane Aparecida Silveira²

\section{RESUMO}

Objetivo: identificar a percepção dos trabalhadores rurais sobre os sinais e sintomas que podem estar relacionados as intoxicações, conhecimento e uso dos Equipamentos de Proteção Individual (EPI) e dos riscos dos agrotóxicos. Metodologia: pesquisa quantitativa, descritiva, epidemiológica, desenvolvida em uma cidade do interior de Minas Gerais com 72 trabalhadores rurais utilizando um instrumento semiestruturado. A análise dos dados foi através de estatística descritiva. Resultados: a maioria dos participantes foi composta por homens, 66 (91,67\%) dos trabalhadores relatam conhecer os EPIs, porém $44(61,11 \%)$ relatam não usar sequer um tipo dos equipamentos necessários, sete (9,72\%) não conheciam nenhum tipo de dano a saúde, 65 (90,28\%) dos entrevistados nunca se intoxicaram, enquanto sete $(9,72 \%)$ já se intoxicaram pelo menos uma vez. Conclusão: são necessárias intervenções visando a redução das intoxicações e a conscientização sobre a utilização dos EPIs assim como dos agravos e danos que o uso de agrotóxicos pode trazer a saúde.

Descritores: Saúde do Trabalhador; Agrotóxicos; Equipamento de Proteção Individual.
${ }^{1}$ Graduada em Enfermagem pela Pontifícia Universidade Católica de Minas Gerais (PUC Minas), Poços de Caldas, MG, Brasil.

${ }^{2}$ Doutora em Enfermagem pela Escola de Enfermagem de Ribeirão Preto da USP (EERPUSP), Ribeirão Preto, SP, Brasil.

\begin{abstract}
Objective: To identify the perception of rural workers of signs and symptoms that may be related poisonings, knowledge and use of Personal Protective Equipment (PPE) and the risks of pesticides. Methodology: Quantitative, descriptive, epidemiological research, developed in a city in Minas Gerais with 72 rural workers using a semi-structured instrument. Data analysis was using descriptive statistics. Results: The majority was made up of men, 66 (91.67\%) of workers report knowing the PPE, but $44(61.11 \%)$ reported not even use a type of equipment needed, $7(9.72 \%)$ did not know any health damage, $65(90.28 \%)$ of respondents never poisoned, while seven $(9.72 \%)$ already were intoxicated at least once. Conclusion: are necessary interventions to reduce poisoning and awareness of the use of PPE as well as the injuries and damage that the use of pesticides can bring health.
\end{abstract}

Descriptors: Occupational Health; Pesticids; Personal Protective Equipment. 


\section{Introdução}

A exposição aos agrotóxicos constitui um grave problema de saúde pública no Brasil, especialmente entre trabalhadores rurais ${ }^{1}$. Fatores como: monitoramento deficitário da exposição ocupacional aos agrotóxicos, elevadas taxas de subnotificação de casos de intoxicação, a não consideração de determinantes sociais e econômicos na avaliação de riscos químicos e a falta de conhecimento dos trabalhadores rurais quanto ao risco da exposição agravam este problema².

A Organização Mundial de Saúde estima que ocorra no mundo, cerca de três milhões de intoxicações agudas por agrotóxicos, sendo $70 \%$ nos países em desenvolvimento como o Brasil, ocorrendo com trabalhadores que tem contato direto ou indireto com esses produtos ${ }^{3}$.

De acordo com dados da Agência Nacional de Vigilância Sanitária, o Brasil está entre os maiores consumidores mundiais de agrotóxicos ${ }^{4}$. Sua utilização descontrolada traz, há várias décadas, incalculáveis prejuízos ao ambiente e ao homem. Os riscos de intoxicação humana acontecem não somente através do trabalho na agricultura, como também, durante a atividade de pulverização, em que acontece uma dispersão destes produtos no ambiente, bem como na alimentação' .

As intoxicações causadas pelos agrotóxicos podem ser agudas, aquelas que os sintomas aparecem subitamente ou crônica, caracterizada por aparecimento tardio, mediante exposição pequena, moderada e contínua. Há ainda sintomas da intoxicação subaguda, associada a uma exposição moderada, os quais são subjetivos e vagos como, por exemplo, dor de cabeça, mal-estar, dor no estômago, fraqueza e sonolência, entre outros ${ }^{5}$.

A literatura relata que, a longo prazo, os agrotóxicos podem causa: patologias de pele, teratogênese, carcinogênese, desregulação endócrina, neurotoxicidade, efeitos na reprodução humana e no sistema imunológico ${ }^{6}$.

A situação se agrava ao se constatar que os trabalhadores rurais não fazem uso dos Equipamentos de Proteção Individual (EPI) adequados ao se exporem aos agrotóxicos, assim se tornando cada vez mais vulneráveis as intoxicações. Além disso, os riscos decorrentes da não utilização de EPI durante as atividades e do desconhecimento de sua importância são subestimados pelos trabalhadores, os quais, sem proteção adequada, se expõem aos riscos de adoecimentos e acidentes ${ }^{7}$.

O fato de que a saúde dos trabalhadores rurais é afetada pela exposição direta aos agrotóxicos aponta a necessidade de medidas para redução de riscos na utilização dos produtos, visando à melhoria na qualidade do trabalho e evitando danos futuros a esses trabalhadores ${ }^{8}$.

É necessário que os profissionais de saúde compreendam que o trabalho é um importante fator determinante da saúde ${ }^{9}$. Esse conhecimento acerca dos agravos de saúde que acometem a população trabalhadora contribui para 0 planejamento de estratégias para lidar com os problemas laborais em todos os níveis de atenção.

Diante do exposto, o objetivo do estudo foi identificar a percepção dos trabalhadores rurais sobre os sinais e sintomas que podem estar relacionados as intoxicações, conhecimento dos riscos dos agrotóxicos e uso dos EPIs.

\section{Metodologia}

Trata-se de uma pesquisa quantitativa, descritiva, de natureza epidemiológica, desenvolvida em uma cidade do interior de Minas Gerais que tem uma economia primária baseada na agricultura, particularmente batata, com alto índice de uso de agrotóxicos.

Fizeram parte deste estudo trabalhadores rurais, adotando-se critérios de inclusão o trabalhador estar envolvido(a) com a agricultura há mais de cinco anos (justifica-se por ser o tempo médio para aparecimento dos primeiros sinais $\mathrm{e}$ sintomas a longo prazo); ser maior de 23 anos de idade (o trabalho com agrotóxicos só é permitido aos maiores de 18 anos mais os cinco anos para o possível aparecimento de sintomas), ter carga horária semanal de 40 horas (por ser trabalho contínuo como rural). Utilizou-se como critério de exclusão, ser caracterizado como trabalho não continuo como rural (carga horária variável menor que 40 horas semanais) ou trabalhar há menos de cinco anos seguidos ou intercalados.

Os participantes foram selecionados mediante sorteio manual, realizado pela pesquisadora, em posse da lista preenchida dos profissionais cadastrados na Estratégia de Saúde da Família como trabalhadores Rurais. Após o sorteio, os mesmos foram contatados no próprio local de trabalho sobre a possibilidade de participarem da pesquisa, bem como questionados quanto ao atendimento dos critérios de inclusão e esclarecidos sobre o objetivo da pesquisa. Dos 100 trabalhadores sorteados, quinze não preenchiam os critérios de inclusão, sete não forma encontrados depois de duas visitas e seis não aceitaram participar da pesquisa, chegando-se a 72 participantes. 
Para a realização deste estudo, foram respeitadas as Diretrizes e Normas Regulamentadoras de Pesquisa envolvendo Seres Humanos, emanadas da Resolução $n^{\circ}$ 466/12, sendo aprovada pelo Comitê de Ética em Pesquisa da Pontifica Universidade Católica de Minas Gerais (CAAE 0004.0.213.000).

Para a coleta de dados os sujeitos da pesquisa foram abordados pelas pesquisadoras em suas residências para responder 0 instrumento de pesquisa. Após serem orientados sobre os objetivos da pesquisa, os participantes que consentiram assinaram o termo de Consentimento Livre e Esclarecido e responderam o questionário.

Foi utilizado um questionário semiestruturado para a coleta de dados primários para caracterização da amostra. Para avaliação da exposição ocupacional aos agrotóxicos, foram coletadas informações sobre a utilização desses produtos, se os indivíduos receberam orientação técnica sobre a utilização dos agrotóxicos, se conhecem e utilizam EPIs preconizados para proteção química. Para a classificação de intoxicação aguda foi solicitado aos trabalhadores que marcassem os sinais e sintomas identificados em até 24 horas após o contato com agrotóxicos. Os sinais e sintomas foram: cefaléia, irritação cutâneo-mucosa, dermatite de contato irritativa ou por hipersensibilização, náusea, vômitos, cólicas abdominais, tontura mais intensa, fraqueza generalizada, parestesia, dispneia, salivação e sudorese aumentadas, hipotensão, arritmias cardíacas, insuficiência respiratória, edema agudo de pulmão, pneumonite química, convulsões, alterações da consciência, choque, coma. Não foi feita a classificação de gravidade.

Para a identificação da intoxicação crônica foi solicitado aos trabalhadores que marcassem os sinais e sintomas identificados em algum momento após trabalharem com agrotóxico. Foram considerados sinais e sintomas imunológicos, hematológicos, hepáticos, neurológicos, malformações congênitas e tumores conforme o Protocolo de Atenção à Saúde dos Trabalhadores Expostos a Agrotóxicos, elaborado pelo Ministério da Saúde ${ }^{10}$.

A análise dos dados foi através de estatística descritiva. A tabulação dos dados e as análises estatísticas realizadas no presente estudo, foram feitas através dos programas Excel 2010 e SPSS 21.0 (Statistical Package for Social Sciences). Foram realizadas análises descritivas para todas as variáveis. Análises de Qui-Quadrado foram realizadas para avaliar a existência de associação não aleatória entre variáveis categóricas.

\section{Resultados}

Foram entrevistados 72 trabalhadores, os quais têm seus dados biopsicossociais apresentados na Tabela 1.

Tabela 1 - Distribuição dos trabalhadores rurais, segundo dados biopsicossociais. Minas Gerais. ( $\mathrm{n}=72)$

\begin{tabular}{c|c|c|c}
\hline \multicolumn{2}{c|}{ Variáveis } & $\begin{array}{c}\text { Total } \\
\mathbf{n}\end{array}$ & $\%$ \\
\hline \multirow{4}{*}{ Sexo } & Feminino & 3 & 4,17 \\
\cline { 2 - 4 } & Masculino & 69 & 95,83 \\
\cline { 2 - 4 } & Total & $\mathbf{7 2}$ & $\mathbf{1 0 0 , 0 0}$ \\
\hline \multirow{4}{*}{ Faixa etária } & 25 a 35 anos & 25 & 34,72 \\
\cline { 2 - 4 } & 35 a 45 anos & 21 & 29,17 \\
\cline { 2 - 4 } & 45 a 60 anos & 26 & 36,11 \\
\cline { 2 - 4 } & Total & $\mathbf{7 2}$ & $\mathbf{1 0 0 , 0 0}$ \\
\hline \multirow{4}{*}{ Escolaridade } & Alfabetizados & 47 & 65,28 \\
\cline { 2 - 4 } & Ensino Fundamental & 19 & 26,39 \\
\cline { 2 - 4 } & Ensino Médio & 6 & 8,33 \\
\cline { 2 - 4 } & Total & $\mathbf{7 2}$ & $\mathbf{1 0 0 , 0 0}$ \\
\hline
\end{tabular}

Fonte: dados da pesquisa.

De acordo com o sexo, $69(95,83 \%)$ dos entrevistados eram homens e apenas três $(4,17 \%)$ mulheres. Quanto à faixa etária, $25(34,72 \%)$ tinham idade compreendida entre 25 a 35 anos. De acordo com a escolaridade, $47(65,28 \%)$ eram apenas alfabetizados, 19 (26,39\%) possuíam Ensino Fundamental e seis (8,33\%) Ensino Médio. 
Tabela 2 - Distribuição dos trabalhadores rurais, segundo exposição aos agrotóxicos, conhecimento e uso de EPIS, efeitos e intoxicações. Minas Gerais. ( $n=72)$

\begin{tabular}{|c|c|c|c|}
\hline \multicolumn{2}{|l|}{ Variáveis } & Total & $\%$ \\
\hline \multirow{4}{*}{$\begin{array}{l}\text { Tempo de } \\
\text { trabalho com } \\
\text { agrotóxicos }\end{array}$} & 5 a 9 anos & 11 & 15,28 \\
\hline & 10 a 14 anos & 5 & 6,94 \\
\hline & Mais de 14 anos & 56 & 77,78 \\
\hline & Total & 72 & 100 \\
\hline \multirow{4}{*}{$\begin{array}{l}\text { Frequência do } \\
\text { trabalho com } \\
\text { agrotóxicos }\end{array}$} & Todos os dias & 4 & 5,56 \\
\hline & Duas vezes por semana & 53 & 73,61 \\
\hline & Uma vez por mês & 15 & 20,83 \\
\hline & Total & 72 & 100 \\
\hline \multirow{6}{*}{ Sobre EPIs } & $\begin{array}{l}\text { Teve treinamento, conhece todos } \\
\text { e faz uso }\end{array}$ & 20 & 27,78 \\
\hline & $\begin{array}{l}\text { Sem treinamento, conhece pela } \\
\text { prática e faz uso }\end{array}$ & 8 & 11,11 \\
\hline & $\begin{array}{l}\text { Teve treinamento, conhece todos, } \\
\text { mas não faz uso }\end{array}$ & 34 & 47,22 \\
\hline & $\begin{array}{l}\text { Não teve treinamento, não conhece } \\
\text { todos, mas faz uso de alguns }\end{array}$ & 4 & 5,56 \\
\hline & $\begin{array}{c}\text { Não teve treinamento, não conhece, } \\
\text { nem faz uso }\end{array}$ & 6 & 8,33 \\
\hline & Total & 72 & 100,00 \\
\hline \multirow{4}{*}{$\begin{array}{l}\text { Identificação de } \\
\text { sinais e sintomas }\end{array}$} & Sinais e/ou sintomas agudos & 24 & 33,33 \\
\hline & Sinais e/ou sintomas crônicos & 10 & 13,89 \\
\hline & Não se aplica & 38 & 52,78 \\
\hline & Total & 72 & 100,00 \\
\hline
\end{tabular}

Fonte: dados da pesquisa.

Do total, $56(77,78 \%)$ trabalham com agrotóxicos há mais de 14 anos, sendo 53 (73,61\%) pelo menos uma vez por semana.

Apesar da maioria dos trabalhadores relatarem conhecer os EPIS, apenas $28(38,89 \%)$ fazem uso de todos os EPIs e somente $54(75 \%)$ trabalhadores receberam orientações sobre o uso dos mesmos. A maioria $(83,33 \%)$ relatou que não faz uso porque os EPIs incomodam.

Dos 72 trabalhadores, $65(90,28 \%)$ trabalhadores rurais disseram ter conhecimento quanto aos danos à saúde.

Em relação à identificação de sinais e sintomas que podem estar relacionados à intoxicação aguda, 24 (33,33 \%) relataram que já identificaram, sendo $10(26,39 \%)$ pelo menos uma vez e cinco $(6,94 \%)$ duas vezes ou mais. Quanto ao procurar atendimento médico devido a intoxicação aguda, dos $24(33,33 \%)$ trabalhadores que já se intoxicaram pelo menos uma vez, 18 (25\%) procuraram atendimento médico, enquanto seis (8,33\%) não procuraram nenhum tipo de atendimento.

Em relação à identificação de sinais e sintomas que podem estar relacionados a intoxicação crônica, 10 (13,89\%) relataram danos à saúde que podem estar relacionados ao uso do agrotóxico.

Não foi constatada associação significativa entre escolaridade e uso de EPI, conhecimento/treinamento sobre EPI e intoxicação. 


\section{Discussão}

A maioria dos trabalhadores do meio rural é formada por homens, maioria também estes que utilizam agrotóxicos ${ }^{11}$. Pessoas de 30 a 44 anos de idade e do contingente de 45 a 59 anos de idade representam os maiores contingentes de indivíduos na agricultura ${ }^{12}$.

A escolaridade é um fator marcante para a saúde do trabalhador rural e para a prevenção da intoxicação. Entretanto, o elevado número de agricultores que têm baixa escolaridade leva a questionar o quanto ele está preparado para entender as instruções da bula do produto, não só em relação à periculosidade, mas também quanto à sua forma de aplicação ${ }^{13}$.

Isso se torna mais grave ao analisar a exposição contínua destes trabalhadores e o fato dos mesmos a entenderem como um fator inerente à sua profissão, preocupando-se mais com a produtividade e a obtenção do lucro mínimo, do que com sua própria saúde ${ }^{14}$.

A questão de conhecer, mas não usar EPI é grave. A análise dos dados mostra que frequentemente o EPI é negligenciado ou é usado inadequadamente, pois em geral é tido pelos agricultores como incômodo e inadequado para o clima quente ${ }^{8}$.

De acordo com um estudo realizado sobre a contaminação por agrotóxicos e uso do EPI, os mesmos são projetados de forma a garantir proteção contra agentes químicos externos, ou seja, para manter certas substâncias "fora" do organismo. As mesmas propriedades físicas e químicas que fornecem aos EPI essa característica de proteção também os transformam, frequentemente, em equipamentos desconfortáveis e/ou inadequados ${ }^{15}$. Esse desconforto no uso pode tornar a utilização de alguns EPIs um verdadeiro incômodo ${ }^{16}$.

Outra questão é que o uso adequado de EPI compreende a utilização de todos os itens que o compõem, chapéu, óculos, máscara, protetor solar, macacão, avental, botas e luvas. Entretanto, o mais comum é usarem em ordem de frequência decrescente: chapéu, seguido de bota, máscara, luva e macacão ${ }^{16}$.

Além da questão da falta de informação em alguns casos, as orientações sobre métodos de uso, manutenção e armazenagem dos EPIs não são validadas nos diversos tipos de situações de trabalho ${ }^{15}$. Em consequência, os métodos prescritos não correspondem aos métodos praticados. Orientações sobre uso do EPI, rótulos e bulas dos agrotóxicos podem não apresentar uma boa legibilidade, com dimensões de letras inadequadas aos usuários. A redação também pode dificultar a comunicação das informações relevantes para o usuário, considerando-se a escolaridade da maioria dos trabalhadores rurais.

É preciso esclarecer e conscientizar através de cursos e palestras quanto aos riscos da exposição dos trabalhadores aos agrotóxicos, assim como exposição de animais e ambiente aos efeitos dos mesmos, pois na maioria das vezes os trabalhadores negam a ocorrência de problemas de saúde relacionados ao uso de agrotóxicos ${ }^{8}$.

Os trabalhadores não se protegem não por não terem conhecimento do risco que correm, mas sim por não acreditarem no mesmo. Sendo assim se torna importante perceber o risco relacionado a uma determinada tarefa, pois pode fazer com que alguma medida de proteção seja tomada por aqueles que a estão realizando, como o uso de algum equipamento de proteção ${ }^{16}$.

Quando percebido sintomas de intoxicação aguda, a atitude mais frequente foi a busca pelo serviço de saúde. Porém, observa-se que o principal entendimento sobre "intoxicação" para a grande maioria dos trabalhadores rurais se refere a situações que necessitam de acompanhamento médico. Ou seja, sintomas mais brandos, como dor de cabeça e enjoo, parecem não ser reconhecido como sintomas de intoxicação, o que demonstra uma naturalização do uso de agrotóxicos ${ }^{17}$, A questão da falta de auto percepção do problema de saúde pode impactar de forma bem expressiva na subnotificação dos $\operatorname{casos}^{18}$.

Entretanto, trabalhadores já internados por intoxicação por agrotóxicos tem 6,03 vezes mais chances de novamente apresentar sintomas comparados àqueles que não vivenciaram esse episódio ${ }^{5}$, seja por viés de informação, seja porque fica mais atento aos sinais.

Quanto à intoxicação crônica percebeu-se a escassez de informações. A percepção dos agricultores sobre os problemas de saúde só apresenta relação com sintomatologias na intoxicação aguda, isso quando fazem essa conexão. Entretanto, a intoxicação crônica é silente, um significante motivador de alerta para a saúde pública ${ }^{16}$. No entanto, há efeitos oncogênicos, cutâneos, oculares, neurológicos e gastrointestinais ${ }^{19}$, além de transtornos mentais ${ }^{20}$. 
Destaca-se que também há despreparo das equipes de saúde para diagnosticar estes problemas, sendo necessária a capacitação desses profissionais quanto ao tema. Apesar da Portaria N ${ }^{\circ} 777 / 2004$, do Ministério da Saúde, definir as intoxicações exógenas, dentre as quais, as causadas por agrotóxicos, como de notificação compulsória segundo o fluxo do Sistema de Informação de Agravos de Notificação (SINAN), ainda não é uma atividade incorporada pelo profissional de saúde ${ }^{10}$.

Além da notificação, o profissional de saúde deve sensibilizar os trabalhadores rurais para a importância do uso correto de EPI, de práticas mais seguras diante da gravidade do emprego indiscriminado e incorreto de pesticidas. Abordar a relação entre as inúmeras patologias e os praguicidas, por meio de intoxicações agudas e crônicas, também pode ser uma estratégia útil, almejando à promoção de saúde e prevenção de agravos ${ }^{8}$.

A equipe de saúde cuidadora necessita aliar assistência e educação em saúde, de forma permanente. Assim, pode-se contribuir na prevenção, atenção, proteção e promoção da saúde, principalmente, de agricultores que utilizam agrotóxicos na lavoura, extensivo aos seus familiares e comunidade ${ }^{21}$.

\section{Considerações Finais}

Apesar da pequena amostra estudada e da impossibilidade de se fazer uma ampla generalização dos resultados deste trabalho, dados preocupantes foram obtidos, os quais necessitam de intervenções. Dados como a quantidade dos trabalhadores rurais que possuem conhecimento sobre os EPIs, mas não usam, além daqueles que não os conhecem e não possuem consciência da importância do seu uso mostram a necessidade de se fazer uma intervenção neste meio.

Outra preocupação é que a maioria dos trabalhadores relata conhecer os efeitos dos agrotóxicos, porém ignora que muitos dos sinais e sintomas apresentados podem estar relacionados ao uso dos agrotóxicos. Isso mostra que, ao contrário do que relataram os trabalhadores rurais entrevistados, os mesmos não possuem conhecimento sobre os danos causados à saúde e até mesmo confundem os sintomas causados pela utilização dos agrotóxicos com qualquer outro tipo de patologia.

Pode-se perceber que a grande maioria dos trabalhadores rurais possui baixa escolaridade, o que faz com que essa classe de trabalhadores, seja desvalorizada e não vista pela sociedade, o que acarreta um sério problema de saúde pública no Brasil, tendo em vista que o analfabetismo está diretamente ligado ao conhecimento sobre agrotóxicos, já que as instruções de uso dos mesmos veem em bulas na embalagem.

Os profissionais de saúde devem trabalhar na conscientização para a prevenção de danos a saúde desses trabalhadores a curto e a longo prazo. É necessário que após a identificação das causas mais comuns, a equipe saiba planejar e atuar afim de que os problemas existentes sejam minimizados tanto quanto a exposição, bem como a prevenção.

\section{Referências}

1. Ferreira de Siqueira D, Moura RM de, Laurentino C, Elizabeth G, Araújo AJ de, Lopes Cruz S. Analysis of rural workers'exposure to pesticides. Rev Bras Em Promoção Saúde. 2013;26(2):182-91.

2. Barbosa R dos S, Silveira HF da, Cerqueira GS, Junior HLR, Carvalho SS de, Alves G rlos S. Exposição ocupacional aos agrotóxicos : um estudo bibliográfico. RevInter Rev Intertox Toxicol Risco Ambient E Soc. 2014;7(1):50-61.

3. International Labor Organization. World Day for Safety and Health at Work: A Background Paper. In: Focus Programme on SafeWork. Geneva: International Labour Office, The World Health Organization; 2005.

4. Cassal VB, Azevedo LF de, Ferreira RP, Silva DG da, Simão RS. Agrotóxicos: uma revisão de suas consequências para a saúde pública. Rev Eletrônica Em Gest Educ E Tecnol Ambient. 2014;18(1):437-45.

5. Mello CM de, Silva LF. Fatores associados à intoxicação por agrotóxicos: estudo transversal com trabalhadores da cafeicultura no sul de Minas Gerais. Epidemiol E Serviços Saúde 2013;22(4):609-20.

6. Begnini S, Taveira ADVA. Agrotoxicos agrícolas: do uso às intoxicações. Rev Ibero-Am Ciênc Ambient. 2014;5(2):86.

7. Martins CL, Jacondino MB, Antoniolli L, Braz DL, Bazzan JS, Guanilo MEE. Equipamentos de proteção individual na perspectiva de trabalhadores que sofreram queimaduras no trabalho. Rev Enferm UFSM 2013; 3(Esp): 668-78

8. Viero CM, Camponogara S, Cezar-Vaz MR, Costa VZ da, Beck CLC. Risk society: the use of pesticides and implications for the health of rural workers. Esc Anna Nery. 2016;20(1):99-105.

9. Ministério da Saúde. Cadernos de Atenção Básica - Saúde do trabalhador [Internet]. Vol. 5. Brasília: Ministério da Saúde; 2002. 68 p. 
10. Ministério da Saúde. Diretrizes para Atenção Integral à Saúde do Trabalhador de Complexidade Diferenciada: Protocolo de Atenção à Saúde dos Trabalhadores Expostos a agrotóxicos. Brasília: Ministério da Saúde; 2006.

11. Moura LTR de, Morais RJL de, Dias ACS, Bedor CNG. Perfil epidemiológico dos casos de intoxicação por agrotóxicos. Rev Enferm UFPE Online. 2014;8(supl. 1):2333-41.

12. Sales EC, Ramos JCL, organizadores. Guia para Análise da Situação de Saúde do Trabalhador - SUS/Bahia. Salvador: DIVAST; 2014. $92 \mathrm{p}$.

13. Silva FM da, Sobrinho LG de A, Coelho DC, Ferreira PM de L, Azevedo PB de. Percepção de risco no uso de agrotóxicos em cinco comunidades rurais no município de Pombal-PB. Rev Verde Agroecol E Desenvolv Sustentável. 2015;9(5):01-9.

14. Rangel C de F, Rosa ACS, Sarcinelli P de N. Uso de agrotóxicos e suas implicações na exposição ocupacional e contaminação ambiental. Cad Saúde Coletiva. 2011;19(4):435-42.

15. Ismael LL, Garcia HRM, Martins WA, Augusto J. Saúde, meio ambiente e segurança do trabalho associado ao uso de agrotóxicos. Rev Verde Agroecol E Desenvolv Sustentável. 2015;10(5):28.

16. Ubessi LD, Ubessi C, Kirchner RM, da Rosa Jardim VM, Fernandes Stumm EM. Use of protective equipment by farmers who use pesticides in relation to health problems. J Nurs UFPE Line. 2015;9(4):7230-8.

17. Germano G de J, Pimentel MS, Machado NG, Mello GJ. Meio ambiente, dialética da agroecologia e hortaliças não convencionais como tema transversal no ensino de ciências. Rev Monogr Ambient. 2015;14:135-46.

18. Brouwer M, Schinasi L, Freeman LEB, Baldi I, Lebailly P, Ferro G, et al. Assessment of occupational exposure to pesticides in a pooled analysis of agricultural cohorts within the AGRICOH consortium. Occup Environ Med. 2016;73(6):359-67.

19. Marangoni SR, Seleghim MR, Santos JAT, Buriola AA, Ballani T da SL, Oliveira MLF de. Intoxications by pesticides recorded at a poisoning control center. J Nurs UFPE Online 2011;5(8):1884-90.

20. Araújo JNG, Greggio MR, Pinheiro TMM. Agrotóxicos: a semente plantada no corpo e na mente dos trabalhadores rurais. Psicol em Rev. 2014;19(3):389-406.

21. Rigotto RM, Vasconcelos DP e, Rocha MM, Rigotto RM, Vasconcelos DP e, Rocha MM. Pesticide use in Brazil and problems for public health. Cad Saúde Pública. 2014;30(7):1360-2.

\section{Cristiane Aparecida Silveira}

Endereço para correspondência - Rua: Pe. Francis Cletus Cox, $n^{\circ} 1661$, Bairro: Jardim Country Club, CEP: 37701-355, Poços de Caldas, MG, Brasil.

E-mail: casilve@yahoo.com.br

Lattes: http://lattes.cnpq.br/8840798305145545

Jéssica Vilela Silva - je-vilela@hotmail.com

Loraine Pires Vilela - loraine_vilela@hotmail.com

Maiara Silva Moraes - maiarahff@hotmail.com

\section{Enviado em 12 de maio de 2016. Aceito em 11 de novembro de 2016.}

